\title{
Study on the measurement method of building decoration bearing capacity based on Improved Genetic Algorithm
}

\author{
Guo Li-mei ${ }^{1}$, Xiao Ai-min ${ }^{2}$ \\ 1. Yibin Vocational \& Technical College, Sichuan 644003, China \\ 2. Yi Bin XinHai Decoration Company, Sichuan 644003, China
}

Keywords: pressure-bearing; building; force analysis

\begin{abstract}
In architectural decoration process, pressure-bearing capacity test is the foundation of design, and is very important. To this end, a pressure-bearing capacity test method in architectural decoration design is proposed based on improved genetic algorithm. The selection, crossover and mutation operators in genetic algorithm are improved respectively. Using its fast convergence characteristics eliminate the pressure movement in the calculation process. The abnormal area of pressure-bearing existed in buildings which can ensure to be tested is added, to obtain accurate distribution information of the abnormal area of pressure-bearing. Simulation results show that the improved genetic algorithm has good convergence, can accurately test the pressure-bearing capacity in architectural decoration.
\end{abstract}

\section{Introduction}

As China's economy continues to develop and the level of urbanization continuously improve, the quality requirements of people on all types of buildings are also increasing [1]. In architectural decoration design process, if the abnormal area of pressure-bearing exists, it will reduce the safety of the architectural decoration and bring security risk for people's life and property [2, 3]. Hence, it needs to accurately test the pressure-bearing situation in architectural decoration design, to take timely and effective measures to eliminate security risks of architectural decoration design. The pressure-bearing area of architectural decoration has discrete and non-linear characteristics, if using traditional genetic algorithm make pressure test, the convergence is slow, and it is easy to fall into "premature" and cannot accurately obtain the pressure-bearing situation of architectural decoration [4-6]. Therefore, how to accurately test the pressure-bearing situation in architectural decoration design, has become a hot problem in the research field of architectural decoration.

\section{The establishment of pressure-bearing capacity test model principle in architectural decoration design}

The establishment of pressure-bearing model of architectural decoration.

In the architectural decoration design process, the pressure of a region can be described using the following formula:

$$
\begin{aligned}
W= & {\left[1-0.175\left(\frac{70^{\circ}-\alpha}{20^{\circ}}\right)\right] \times } \\
& {\left[1-0.07\left(\frac{R}{400 t}\right)\right]\left(0.3 v \frac{t^{2}}{R^{2}}\right) }
\end{aligned}
$$

In the above formula, $\mathrm{W}$ indicates that the subjected static pressure of this area, $\mathrm{v}$ represents the elastic deformation volume of per unit of the architectural decoration materials in this area, $\mu_{s}$ represents the Poisson's ratio of the architectural decoration materials, $R$ represents the critical radius of architectural decoration, $t$ indicates that the thickness of the architectural decoration materials in this area, $\alpha$ represents the angle of the pressure-bearing in this area.

Here, the compressive property of the architectural decoration area can be described using the 
following formula:

$$
\begin{aligned}
V= & \frac{E_{s} t}{R_{\text {eff }}}\left[\frac{m^{4}}{\left(n_{m}^{2}+\left(m^{2} / 2\right)-1\right)\left(n_{m}^{2}+m^{2}\right)^{2}}\right]+ \\
& \frac{\left(n_{m}^{2}-1\right) E_{s} I}{R_{e f f}^{3} L_{s}}
\end{aligned}
$$

In the above formulas,

$$
\begin{aligned}
& \mathrm{R}_{\mathrm{e} f f}=R / 2(1+\sin \alpha) \\
& L_{s}=2 R \cos \alpha \\
& m=\pi R_{\text {eff }} / L_{s}
\end{aligned}
$$

In the above formula, $\mathrm{L}$ represents the sectional area of the architectural decoration materials.

Using the following formula can calculate the maximum pressure-bearing value of this architectural decoration area:

$$
\varsigma_{\text {von-mises }}=\sqrt{\frac{1}{2}\left(\varsigma_{1}-\varsigma_{2}\right)^{2}+\left(\varsigma_{2}-\varsigma_{3}\right)^{2}+\left(\varsigma_{3}-\varsigma_{1}\right)^{2}}
$$

If exceeds the pressure-bearing value of architectural decoration, it will result in lower safety performance of architectural decoration, or even collapse.

Pressure-bearing function of architectural decoration under improved Genetic Algorithm.

The use of improved genetic algorithm test the pressure-bearing area of architectural decoration. In the case of that the above constraints can be met, to the total elastic deformation quantity of architectural decoration can be obtained in architectural design process.

Supposing that there are $m$ pressure-bearing areas on the architectural decoration material of a buildings, the objective function can be expressed using the following equation:

$$
\begin{aligned}
& \max f\left(y_{1}, y_{2}, \ldots y_{m}\right)=\sum_{i=1}^{m} L_{i} t_{i} v_{i} \\
& \sum_{i=1}^{m} L_{i} t_{i} v_{i} \leq c \\
& y_{i} \in\{0,1\}, i \in(1, m)
\end{aligned}
$$

In the above formula, $i$ represents the point of pressure-bearing of $i$-th place in the architectural decoration; $L_{i}$ indicates the cross-sectional area of $i$-th place in the area of architectural decoration; $v_{i}$ represents the elastic deformation quantity per unit volume of the $i$-th place in the area of the architectural decoration materials; $y_{i}$ represents the decision variables from 0 to 1 , and 0 is the concrete material, 1 is the reinforced material; $c$ represents the pressure-bearing of the architectural decoration in a whole building;

\section{Variables selection of architectural decoration pressure-bearing model.}

There are many factors affect the quality of architectural decoration, the principal factors include the intensity level of decorative material, the cross-sectional area of load-bearing walls, etc. in order to simplify the calculation, the parameters which played the role of control for architectural decoration pressure-bearing are selected to make as variables, other factors which has not effects can be considered as constant. Since the architectural decoration materials will produce deformation when subjected to pressure, when its deformation quantity exceeds the maximum deformation quantity it will occur collapse, therefore, the deformation quantity of area, thickness and unit volume in architectural decoration area are selected as design variables.

Constraints of the pressure-bearing capacity tests model of architectural decoration.

The characteristics of the loading force of architectural decoration in various stages of loading is different, therefore, it needs to analyze the loading force of architectural decoration in various stage. 
In order to protect the safety of architectural decoration, the following stress constraints of architectural materials in the pressure-bearing area of architectural decoration:

For the uncracked area of architectural decoration materials:

$\sigma_{k c}+\sigma_{p t} \leq 0.5 f_{c k}$

In the above formula, $\sigma_{k c}$ represents the normal direction tensile stress of architectural decoration materials, it can be represented by the following formula:

$\sigma_{k c}=\frac{M_{g 1}}{W_{n s}}+\frac{M_{k}-M_{g 1}}{W_{o s}}$

In the above formula, $M_{k}$ represents bending moment.

$\sigma_{p t}$ represents the normal direction tensile stress of architectural decoration materials, it can be represented by the following formula:

$$
\sigma_{p t}=\frac{N_{p}}{A_{n}}-\frac{M_{p}}{W_{n s}}
$$

The maximum tensile stress of the architectural decoration materials in the pressure-bearing area

For the uncracked area of architectural decoration materials,:

$\sigma_{p e}+\sigma_{p} \leq 0.65 f_{p k}$

In the above formula, $\sigma_{p e}$ represents the effective prestress of the architectural decoration materials in this area. $\sigma_{p}$ represents the tensile stress generated by the architectural decoration materials in this area, it can be represented by the following formula :

$\sigma_{p}=\alpha_{E P} \sigma_{k}$

Where, $\sigma_{k}=\frac{M_{g 1} e_{n}}{I_{n}}+\frac{\left(M_{k}-M_{g 1}\right) \mathrm{e}_{o}}{I_{o}}$

In the above formula, $e_{n}$ is the distance between the center of a circle of the architectural decoration materials to the section of the area, $\mathrm{e}_{o}$ indicates the distance between the center of a circle of the architectural decoration materials to the exchange axis, $e_{n}=y_{n x}-\alpha_{i}, e_{o}=y_{o x}-\alpha_{i} . \sigma_{k}$ represents the normal direction tensile stress in the center of gravity of architectural decoration materials, $\alpha_{E P}$ represents the elastic modular ratio of different architectural decoration materials.

\section{Simulation results and analysis}

In order to verify the effectiveness of the proposed method, it needs an experiment. The experiment are conducted in matlab environment, and is run 200 times respectively. Population size is 300 , the evolution is set to 160 generations to stop.

In this paper, the improved and traditional genetic algorithm are respectively made simulation experiment, the results are obtained shown in Table 1:

Table 1 experimental results of different algorithms

\begin{tabular}{ccccc}
\hline algorithm & $\begin{array}{c}\text { The total } \\
\text { pressure value }\end{array}$ & $\begin{array}{c}\text { Total elasticity } \\
\text { variable }\end{array}$ & $\begin{array}{c}\text { Pressure point } \\
\text { number }\end{array}$ & $\begin{array}{c}\text { The pressure on } \\
\text { the overall } \\
\text { construction } \\
\text { materials }\end{array}$ \\
\hline $\begin{array}{c}\text { Traditional } \\
\text { algorithm } \\
\begin{array}{c}\text { The proposed } \\
\text { algorithm }\end{array}\end{array}$ & 9824 & 8123 & 200 & 10000 \\
\hline
\end{tabular}


It can be seen from Table 1, the accuracy of using the improved genetic algorithm for pressure-bearing capacity test is higher than traditional genetic algorithm.

The convergence curves of using traditional algorithm and improved algorithm are shown in figure 1:

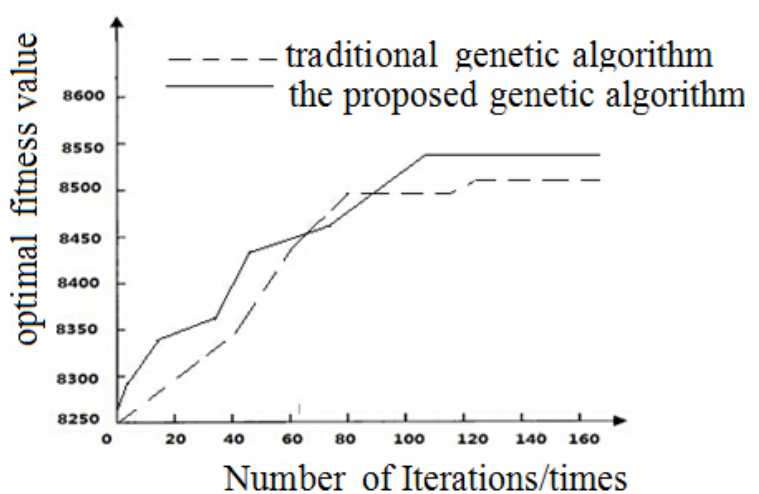

Figure 1 The convergence curves of traditional algorithm and improved algorithm

It can be seen from Figure 1, the improved genetic algorithm in this paper has better convergence and better fitness, and it fully embodies the advantages of the proposed algorithm.

\section{Conclusion}

In this paper, a pressure-bearing capacity test method in architectural decoration design is proposed based on improved genetic algorithm. The selection, crossover and mutation operators in genetic algorithm are improved respectively. Using its fast convergence characteristics eliminate the pressure movement in the calculation process. The abnormal area of pressure-bearing existed in buildings which can ensure to be tested is added, to obtain accurate distribution information of the abnormal area of pressure-bearing. Simulation results show that the improved genetic algorithm has good convergence, can accurately test the pressure-bearing capacity in architectural decoration.

\section{References}

[1] SMITH S J, SUTCLIFFE B T. The Development of Computational Chemistry in the United Kingdom [M]. Reviews in Computational Chemistry. John Wiley \& Sons, Inc. 2007: 271-316.

[2] D P Jones,J E Holliday. Elastic-Plastic analysis of the PVRC burst test with comparison to the ASME code primary stress limits [J]. Journal of Pressure Vessel echnology, ASME 2000(122):146-151

[3] Lin S P,Chang Y W.A novel framework for multilevel routing cons-idering routability and performance[C]PPProc. of Int. Conf. Computer-Aided Design, November 2002:44-50.

[4] Zhang Yuming, Meng Shaoping, Qiu Ronggen, et al. Study on Prestressing Construction Questions of the Large Platform of Nanjing Olympic Sports Center [J]. Construction technology, 2005, 34(7):13-15

[5] D.Hu, X.Tian. A Multi-Directions Algorithm for Edge Detection Based on Fuzzy Mathematical Morphology[C]. Proceeding of the $16^{\text {th }}$ International conference on Aritificial Reality and Telexistence-Workshops(ICAT06), IEEE, 2006, 11 : 361-364.

[6] K. Hirakawa, T.W. Parks, Image Denoising for Signal-Dependent Noise [J]. IEEE ICASSP, 2005, 2:29-32. 